\title{
Simple Bone Cyst and Fibrous Dysplasia Occurring Simultaneously in Both Mandibles: Case Report
}

\author{
Hong-Soon Kim, Chan-Jong Song, Dong-Ju Seol, Jae-Wook Lee, Baek-Soo Lee, \\ Yong-Dae Kwon, Joo-Young Ohe, Jung-Woo Lee, Byung-Joon Choi
}

Department of Oral and Maxillofacial Surgery, Kyung Hee University School of Dentistry, Seoul, Korea

\begin{abstract}
Fibrous dysplasia is a benign fibro-osseous lesion wherein normal bone is replaced with an excessive proliferation of cellular fibrous connective tissue intermixed with irregular bony trabeculae. Fibro-osseous lesions like fibrous dysplasia are often associated with non-epithelial cysts, such as simple bone cyst. The etiologic and pathogenic relationships between fibrous dysplasia and simple bone cyst have not been conclusively established. Nonetheless, the mechanism of cyst formation in fibro-osseous lesion associated with simple bone cyst can be said to differ from that of the typical simple bone cyst of the jaws. This article reports a case of bilateral lesions including fibrous dysplasia and simple bone cyst on each site and reviews the pathogenesis of cyst formation in the fibro-osseous lesion.
\end{abstract}

Key Words: Fibro-osseous lesion; Fibrous dysplasia; Simple bone cyst

\section{Introduction}

Fibrous dysplasia is a metabolic bone disease classified as a benign fibro-osseous lesion. Normal osseous tissue is replaced by abnormal fibrous tissue and is gradually changed into irregular trabeculae ${ }^{1)}$. Although the cause of fibrous dysplasia has yet to be identified, it is related to GNAS1 mutation ${ }^{2}$ showing three types: contraction only in single bone depending on the period of mutation; contraction by multiple lesions ${ }^{3)}$, and; case accompanying endocrinopathy ${ }^{4}$. About $80 \sim 85 \%$ of fibrous dysplasia cases have the type of monostotic contraction and usually develop during the teenage years. It occurs in both males and females, although females tend to record a higher incidence rate these days. Generally, when it occurs in the jawbones, the rate is double in the maxilla than that in the

Corresponding Author: Byung-Joon Choi

Department of Oral and Maxillofacial Surgery, Kyung Hee University School of Dentistry, 1 Hoegi-dong, Dongdaemun-gu, Seoul 130-701, Korea

TEL : +82-2-958-9440, FAX : +82-2-966-4572, E-mail : sjnb2@khu.ac.kr

Received for publication April 11, 2013; Returned after revision May 30, 2013; Accepted for publication June 7, 2013

Copyright $\odot 2013$ by Korean Academy of Dental Science

(c) This is an open access article distributed under the terms of the Creative Commons Attribution Non-Commercial License (http:/ /creativecommons.org/licenses / by-nc/3.0) which permits unrestricted non-commercial use, distribution, and reproduction in any medium, provided the original work is properly cited. 
mandible, frequently occurring in the posterior teeth. It accompanies painless swelling that slowly gets bigger. Radiologically, it typically shows 'ground-glass' pattern because of the trabecula that had insufficient calcification, and the boundary with surroundings are not clear. In the early stages, radiolucent lesions are observed; as lesions develop, however, they are mixed with radiopaque lesions ${ }^{1,5}$. There were some studies on the correlation between fibrous dysplasia and simple bone cyst as well as various claims regarding its pathogenesis ${ }^{6,7)}$.

Therefore, we report a case wherein simple bone cyst and fibrous dysplasia develop on both sides of the mandible and present a literature review.

\section{Case Report}

When one female patient, aged 21, visited our hospital for the first time, she had no symptoms, e.g., pain or aesthetic, functional problems and anamnesis of injury. There were no specific findings in intra-oral and extra-oral examination, and all mandible teeth tested positive on the electric pulp test. Neither was there any abnormal sensation.

On the panoramic picture and periapical radiograph of \#34 and \#37, unicystic radiolucent lesion was observed in the posterior area bone of the left mandible. Its border was relatively clear, and it had a shell shape. The loss of alveolar lamina dura was observed in \#35, 36, and 37, but not clear root resorption or tooth displacement (Figs. 1, 2). Upon additional cone beam computed tomography (CBCT), buccal and lingual cortical bones were found to be thin, with the mandibular canal displaced downward (Fig. 3). The lesion in the posterior area of the left mandible was tentatively diagnosed as keratocystic odontogenic tumor.

In the posterior area of the right mandible throughout \#44 47, radiolucent lesions and radiopaque lesions were mixed, and their border was not clear. The lesion in the posterior area of the right mandible was tentatively diagnosed as fibrous dysplasia (Figs. 1,3).

Based on the tentative diagnoses, we planned

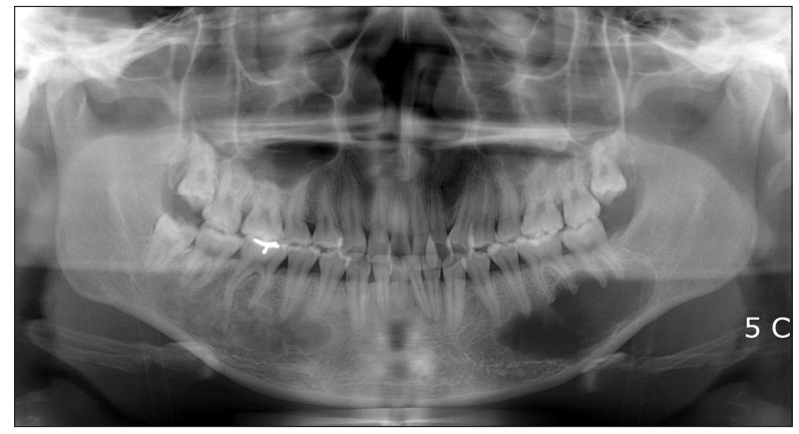

Fig. 1. The panoramic view at the first visit shows well-defined unilocular radiolucent lesion on the \#34 37 area with scalloped and corticated margin. The lesion in the posterior area of the right mandible demonstrates poorly defined lesion with mixed radiodensity.

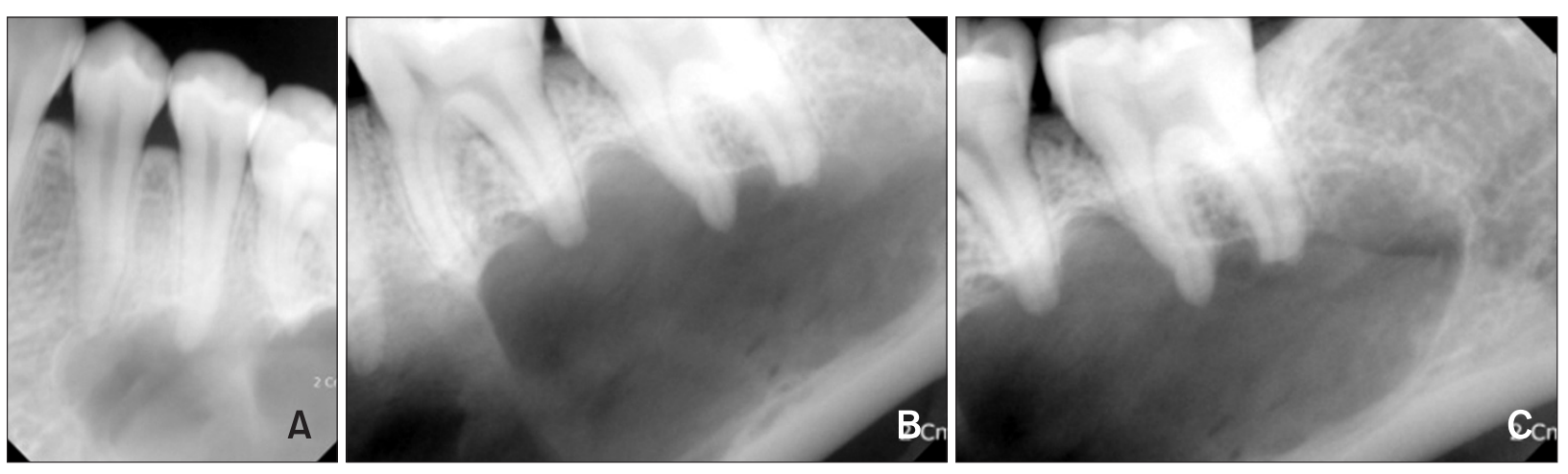

Fig. 2. (A C) The periapical views at the first visit show loss of lamina dura on \#35 37, no definite external root resorption, and tooth displacement on \#34 37. 

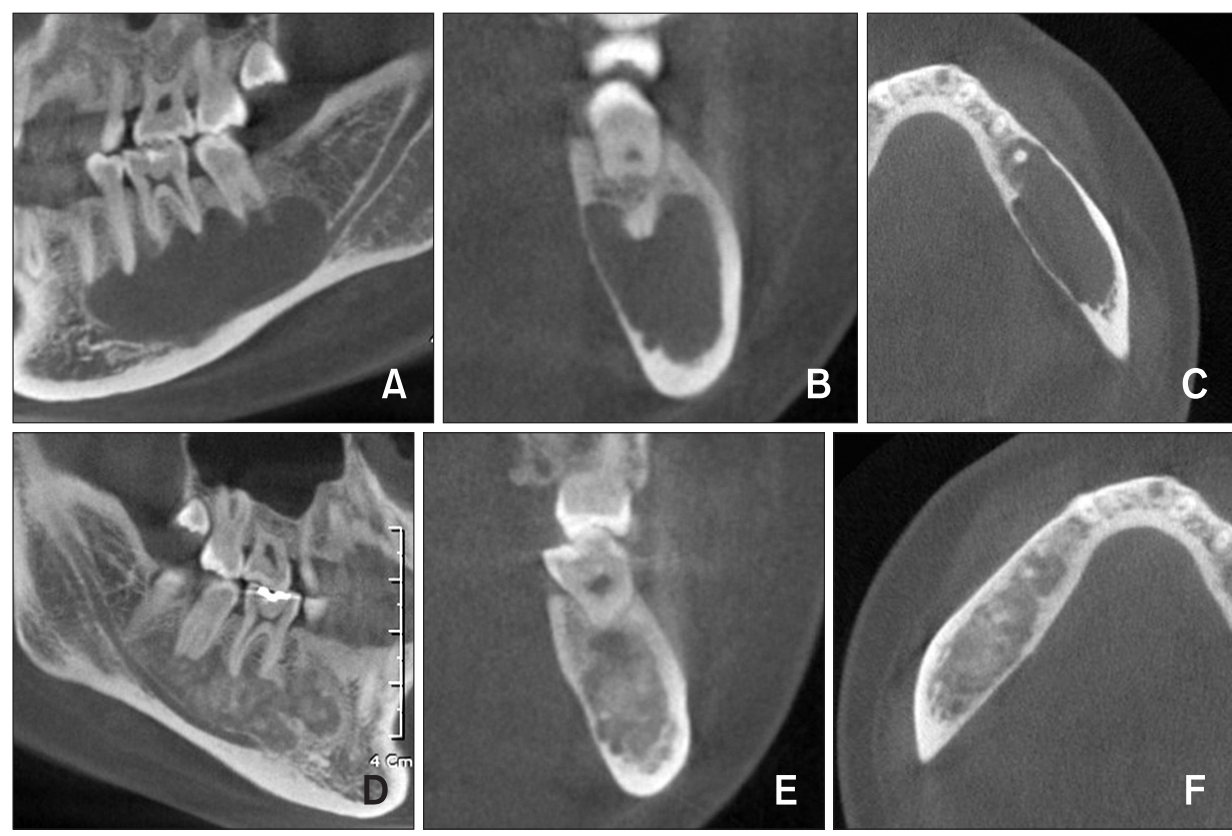

Fig. 3. The computed tomography scan at the initial visit shows in the left mandible $(\mathrm{A} \sim \mathrm{C})$ monolocular lesion with the thinning of both buccal and lingual cortical plates and inferior displacement of the mandibular canal. The lesion of the right mandible $(D \sim F)$ demonstrates poorly demarcated radiopaque lesion with various internal densities.

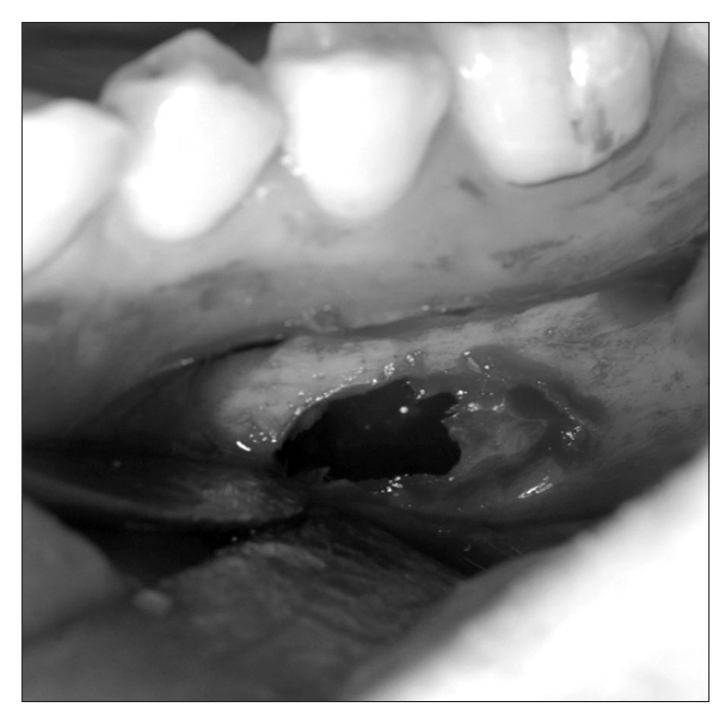

Fig. 4. The inside of the left mandibular lesion was empty with no fluid and lining, surrounded only by a hard bony wall.

surgical resection for the lesion in the posterior area on the left and incision biopsy for the lesion in the posterior area on the right under general anesthesia. The inside of the lesion in the posterior area on the left was empty, and unusual exudation or cyst wall or unusual surrounding membrane or soft tissue was not found (Fig. 4). Therefore, our diagnosis was simple bone cyst, and we performed suturing after confirming that the inside of the cavity was filled up with blood. For the lesion in the posterior area on the right, we performed incisional biopsy and finished by suturing.

Through the histopathological test of tissue extracted from the posterior area on the right, we could find woven bone separated irregularly here and there based on loosely arranged cellular fibrous ground. There were no osteoblasts around the new bone, and it looked like new bone was mixed with normal bone since no border or capsule was seen. The whole appearance was shaped like 'Chinese script writing,' a typical characteristic of fibrous dysplasia (Fig. 5).

After considering the clinical and radiological examinations and histopathological findings together, we finally diagnosed the lesion in the posterior area on the right as fibrous dysplasia and the lesion in the posterior area on the left as simple bone cyst.

In the radiological examination conducted when the patient revisited 6 months later, there was no unusual change in the posterior area of the right mandible. Note, however, that the markedly increased radiopacity of the lesion in the posterior area of the left mandible confirmed that it was in 

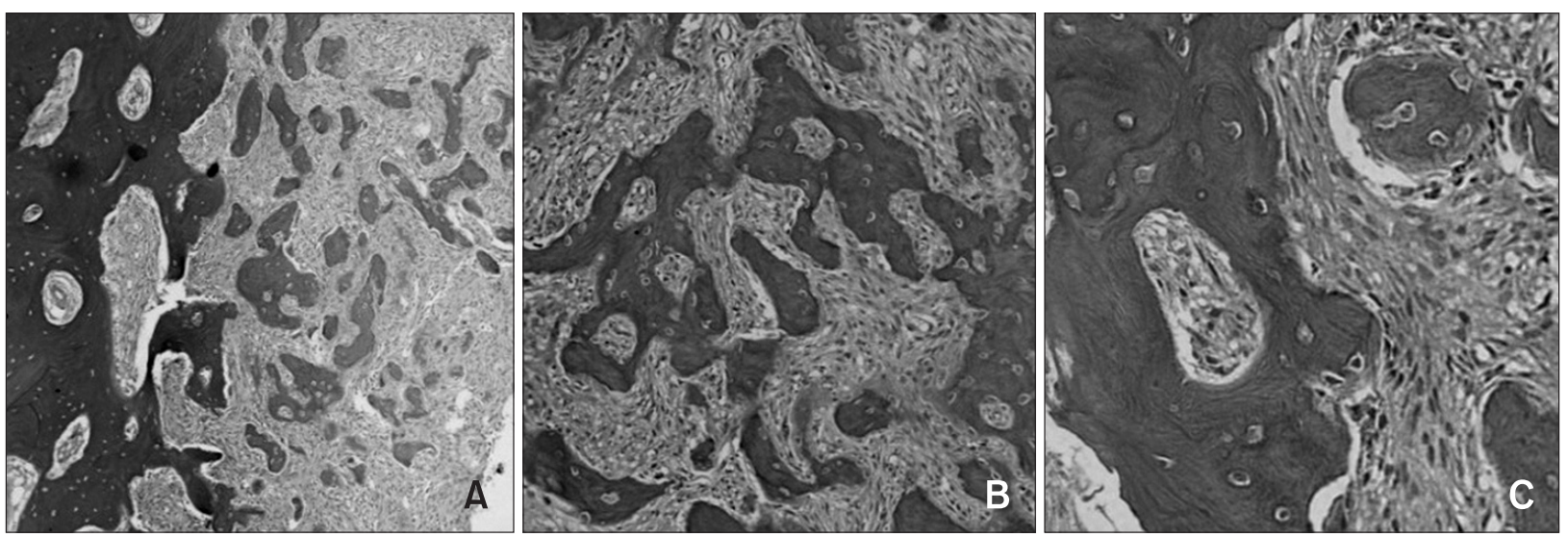

Fig. 5. Histological section (H\&E staining). The low-power photomicrograph of the right mandibular lesion shows irregularly shaped trabeculae of woven bone in fibrous stroma. The high-power photomicrograph reveals no osteoblastic rimming (low-, medium-, high-power) (A: ×10, B: ×20, C: ×40).

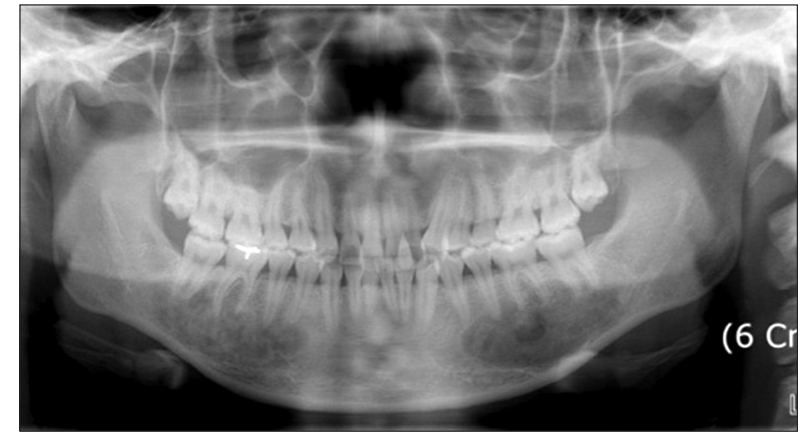

Fig. 6. The panoramic view 6 months after surgery shows increased radiodensity in the left mandible.
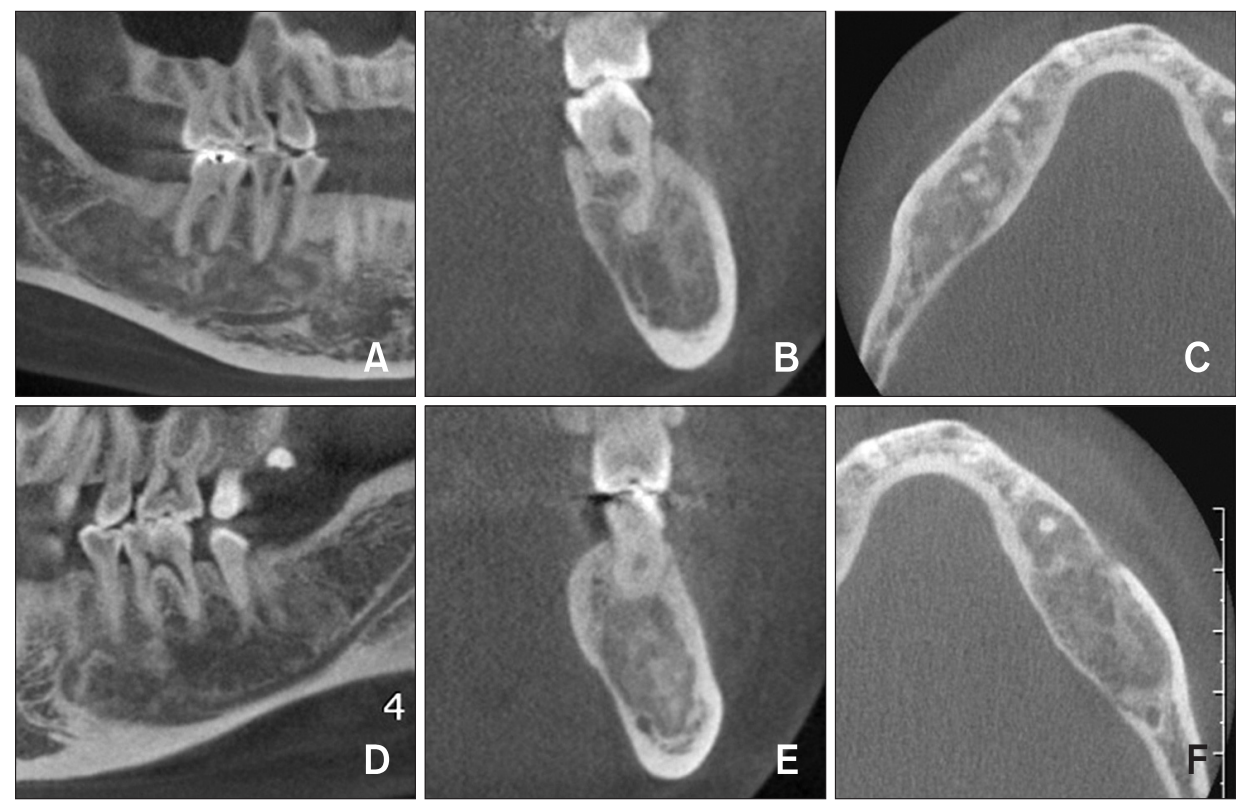

Fig. 7. Left mandible $(D \sim F)$, Right mandible $(A \sim C)$. The computed tomography scan 6 months after surgery shows in the left mandible newly formed tra-beculae within the cavity and unre-markable radiographic cha-nges in the right mandible. 
multinucleated giant cell and woven bone based on the histopathological examination ${ }^{19}$. Since these features were not observed in this case, aneurysmal bone cyst was excluded in the diagnosis.

Simple bone cyst means a cavity within bone that is sometimes filled with liquid and simply surrounded by bone without epithelial lining or infection $^{20)}$. It was first called simple bone cyst by Lucas $^{21)}$ in 1929 and later called in many different ways such as traumatic bone cyst, solitary bone cyst, unicameral bone cyst, hemorrhagic bone cyst, or extravasation bone cyst. This implies that its pathogenesis has not yet been identified exactly. Usually painless clinically, it is sometimes found from the radiograph shot for another treatment purpose in a hospital. Saito et al. ${ }^{16)}$ reported a $1.2 \%$ prevalence rate.

According to Shigematsu et al. ${ }^{22)}$ and Rubin and Murphy $^{23)}$, simple bone cyst usually occurs during teenage years, and $50 \%$ of cases have anamnesis of injury; it is generally unicystic and is not directly connected with the area with maxilla tooth germ. They reported that there was no tendency according to gender, and that it was usually healed after the inside of the cavity was filled with blood after curettage.

Radiologically simple bone cyst is said to take on radiolucency whose border is relatively clear and shaped like scallop. The scalloped shape varies ${ }^{24)}$; root resorption or osseous expansion is also reported ${ }^{25)}$.

According to Saito et al. ${ }^{16}$, clinical, radiological, and histopathological findings in young people and old people are slightly different. They said that painless, radiolucent lesion with minimal osseous expansion was observed in young people as a form wherein the border was relatively clear or scallop-like border was not clear (osteosclerotic bone destruction whose border was clear was not observed). Moreover, the lamina dura of the involved tooth was normal, and there was no tooth displacement. On the whole, the surgical approach got rid of radiolucent lesions immediately, and a newly formed wound promoted bone healing. Of course, simple bone cyst could heal on its own without surgical treatment.

When simple bone cyst develops in old people, it is said to be prone to occur in females; multiple and radiopacity found in cement-osseous dysplasia or loss of lamina dura could also be observed additionally. The formation of this radiolucency could be due to the increase in cyst size; when simple bone cyst persists, the erosion of lamina dura exposes the tooth root, thereby promoting the formation of cement ${ }^{16)}$. Tomita et al. ${ }^{25)}$ also reported that the lamina dura of the involved tooth was more likely to be eroded in atypical simple bone cyst with radiopacity.

There was a report on the case wherein simple bone cyst or aneurysmal bone cyst did not meet the histopathological diagnosis criterion for fibroosseous lesion; this was called non-specific cystic degeneration ${ }^{6,26-28)}$.

In particular, the reason fibro-osseous lesions occur in relation to non-epithelial lining has yet to be identified. Lucas ${ }^{21)}$ suggested the degeneration of connective tissue as the reason for the formation of microcyst. Fisher ${ }^{26)}$ said that fibro-osseous lesions developed secondarily, and that it was due to the healing process of simple bone cyst.

There are many studies reporting that the pathogenesis of simple bone cyst that developed in relation to fibro-osseous lesions was different from general simple bone cyst. Note, however, that Melrose et al. ${ }^{14)}$ reported a case wherein florid osseous dysplasia (renamed 'florid cementoosseous dysplasia' by Waldron ${ }^{29}$ ) developed with simple bone cyst at the same time. They explained that many expansions of capillary were observed in lesions, and that the bone formed in a disorderly manner based on this hindered the circulation of interstitial fluid and consequently induced the formation of cyst.

Higuchi et al. ${ }^{12)}$ reported the case of 4 local cemento-osseous dysplasias separated in simple 
bone cyst. According to them, this showed that cemento-osseous dysplasia developed before the formation of cyst. Simple bone cyst related to cemento-osseous dysplasia was different from the typical pathogenesis of a simple bone cyst assumed to develop from intra-medullar bleeding. Horner and Forman ${ }^{13)}$ also supported this theory.

Miyauchi et al. ${ }^{15)}$ also claimed that simple bone cyst related to cemento-osseous dysplasia had different disease etiology from general simple bone cyst, in reporting the case wherein florid cemento-osseous dysplasia was formed together with simple bone cyst. They reported active capillary network in the medullary cavity of bone located between trabecula formed around the cyst cavity and increasing fibrous tissues. This is consistent with the histopathological findings reported by Melrose et al. ${ }^{14}$.

According to Hara et al. ${ }^{7}$, the formation of cyst lesion seemed to be caused by an aberration in the formation and growth of fibrous dysplasia. The fact that it was prone to occur in females when mixed with radiopacity showed possible connection with the hormone factor in its pathogenesis ${ }^{14,16,30)}$.

Although this study has a limit in assuming the connection with fibrous osseous lesions since biopsy--which requires enough surrounding bones-had not been conducted in case of simple bone cyst in the posterior area of the left mandible. Since the posterior area of the right mandible clearly had lesion of fibrous dysplasia, however, the lesion in the posterior area of the left mandible could be said to be simple bone cyst related to fibrous dysplasia as well as general simple bone cyst.

Simple enucleation is known to be enough to promote blood filling as well as verification for the treatment of simple bone cyst because its prognosis is fine ${ }^{5)}$. When it accompanies fibro-osseous lesion, however, continuous observation is needed. In case of relatively large simple bone cyst encompassing premolar to mandibular angle, Hara et al. ${ }^{7}$ performed primary marsupialization, curettage 18 months later, and plastic surgery 12 months later for the improvement of facial asymmetry. In the case of simple bone cyst accompanying florid cementoosseous dysplasia, Wakasa et al. ${ }^{17)}$ performed primary enucleation and found that the cyst lesion was removed six months later. About 3 years later, however, the lesion recurred. Thus, enucleation was performed again. Similarly, Saito et al. ${ }^{16)}$ performed secondary surgery when they detected a recurrence 8 years later in the case of simple bone cyst related to florid cemento-osseous dysplasia.

Moreover, in this case, which is highly likely to be related to fibro- dysplasia, the possibility of recurrence of lesion is higher than general simple bone cyst, and additional plastic surgery could be needed when bone healing similar to fibrous dysplasia proceeds.

Since the prognosis of simple bone cyst accompanying fibro-osseous lesions could be different from general simple bone cyst, more accurate diagnosis and surgery process are required.

\section{References}

1. White SC, Pharoah MJ: Oral radiology: principles and interpretation. 5th ed. St. Louis (Mo): Mosby; 2004. 485-515.

2. Jundt G: Fibous dysplasia. In: Barnes L, Evenon JW, Reichart P, Sidransky D, eds. Pathology and genetics of head and neck tumours. World Health Organization classification of tumours. Lyon: IARC Press; 2005. 321.

3. Lustig LR, Holliday MJ, McCarthy EF, Nager GT. Fibrous dysplasia involving the skull base and temporal bone. Arch Otolaryngol Head Neck Surg. 2001; 127: 1239-47.

4. Waldron CA. Fibro-osseous lesions of the jaws. J Oral Maxillofac Surg. 1993; 51: 828-35.

5. Neville BW, Damm DD, Allen CM, Boyquot JE: Oral and maxillofacial pathology. 2nd ed. Philadelphia: W.B. Saunders; 2002.

6. Ferretti C, Coleman H, Dent M, Altini M. Cystic degeneration in fibrous dysplasia of the jaws: a 
case report. Oral Surg Oral Med Oral Pathol Oral Radiol Endod. 1999; 88: 337-42.

7. Hara H, Ohishi M, Higuchi Y. Fibrous dysplasia of the mandible associated with large solitary bone cyst. J Oral Maxillofac Surg. 1990; 48: 88-91.

8. Buraczewski J, Dabska M. Pathogenesis of aneurysmal bone cyst. Relationship between the aneurysmal bone cyst and fibrous dysplasia of bone. Cancer. 1971; 28: 597-604.

9. El Deeb M, Sedano HO, Waite DE. Aneurysmal bone cyst of the jaws. Report of a case associated with fibrous dysplasia and review of the literature. Int J Oral Surg. 1980; 9: 301-11.

10. Oliver LP. Aneurysmal bone cyst. Report of a case. Oral Surg Oral Med Oral Pathol. 1973; 35: 67-76.

11. Ackermann GL, Altini M. The cementomas--a clinicopathological re-appraisal. J Dent Assoc S Afr. 1992; 47: 187-94.

12. Higuchi Y, Nakamura N, Tashiro H. Clinicopathologic study of cemento-osseous dysplasia producing cysts of the mandible. Report of four cases. Oral Surg Oral Med Oral Pathol. 1988; 65: 339-42.

13. Horner K, Forman GH. Atypical simple bone cysts of the jaws. II: a possible association with benign fibro-osseous (cemental) lesions of the jaws. Clin Radiol. 1988; 39: 59-63.

14. Melrose RJ, Abrams AM, Mills BG. Florid osseous dysplasia. A clinical-pathologic study of thirty-four cases. Oral Surg Oral Med Oral Pathol. 1976; 41: 6282.

15. Miyauchi M, Ogawa I, Takata T, Ito H, Nikai H, Ijuhin N, Tanimoto K. Florid cemento-osseous dysplasia with concomitant simple bone cysts: a case in a Japanese woman. J Oral Pathol Med. 1995; 24: 285-7.

16. Saito Y, Hoshina Y, Nagamine T, Nakajima T, Suzuki M, Hayashi T. Simple bone cyst. A clinical and histopathologic study of fifteen cases. Oral Surg Oral Med Oral Pathol. 1992; 74: 487-91.

17. Wakasa T, Kawai N, Aiga H, Kishi K. Management of florid cemento-osseous dysplasia of the mandible producing solitary bone cyst: report of a case. J Oral Maxillofac Surg. 2002; 60: 832-5.

18. Makek MS. So called "fibro-osseous lesions" of tumorous origin. Biology confronts terminology. J Craniomaxillofac Surg. 1987; 15: 154-67.

19. Sciubba J, Fantasia JE, Kahn LB: Tumors and cysts of the Jaw. Atlas of tumor pathology. Third series, fasc. 29. Washington, D.C.: Armed Forces Institute of Pathology; 2001. 171.

20. Rushton MA. Solitary bone cysts in the mandible. Br Dent J. 1946; 81: 37-49.

21. Lucas RB. Pathology of tumours of the oral tissues. Edinburgh: Churchill Livingstone; 1976. 390.

22. Shigematsu H, Fujita K, Watanabe K. Atypical simple bone cyst of the mandible. A case report. Int J Oral Maxillofac Surg. 1994; 23: 298-9.

23. Rubin MM, Murphy FJ. Simple bone cyst of the mandibular condyle. J Oral Maxillofac Surg. 1989; 47: 1096-8.

24. Shear M, Speight PM: Cysts of the oral and maxillofacial regions. 4th ed. Oxford, Ames, Iowa: Blackwell Pub; 2007.

25. Tomita S, Fujita M, Yasutomi Y, Aoyama M, Imagawa H, Furuki Y, Sadanari T, Tanimoto K, Uemura S, Wada T. A radiographic study of simple bone cyst. Shika Hoshasen. 1989; 29: 51-62.

26. Fisher AD. Bone cavities in fibro-osseous lesions. Br J Oral Surg. 1976; 14: 120-7.

27. Obwegeser HL, Freihofer HP Jr, Horejs J. Variations of fibrous dysplasia in the jaws. J Maxillofac Surg. 1973; 1: 161-71.

28. Simpson AH, Creasy TS, Williamson DM, Wilson DJ, Spivey JS. Cystic degeneration of fibrous dysplasia masquerading as sarcoma. J Bone Joint Surg Br. 1989; 71: 434-6.

29. Waldron CA. Fibro-osseous lesions of the jaws. J Oral Maxillofac Surg. 1985; 43: 249-62.

30. Zegarelli EV, Kutscher AH, Napoli N, Iurono F, Hoffman P. The cementoma. A study of 230 patients with 435 cementomas. Oral Surg Oral Med Oral Pathol. 1964; 17: 219-24. 